\title{
Optimal Scheduling of Combined cooling heating system Shulei DENG
}

\author{
Chong Qing Electric Power college, Chongqing 400053, China \\ 330355502@qq.com
}

Keywords: combined cooling heating; optimal scheduling; renewable energy; tradable green certificate

\begin{abstract}
Due to the fossil fuel crisis, combined cooling heating and power (CCHP) systems which can jointly supply electricity and hot/cold have become the mainstream of energy generation technology. In this paper, tradable green certificate(TGC) mechanism is firstly introduced to operation of CCHP system, and the impacts of tradable green certificate on the scheduling of CCHP system are studied. Based on the probability distribution of wind speed, an economic scheduling model is proposed, which considers the uncertainties of renewable energy and tradable green certificate price as well as the constraints of secure operation of power system and pipe networks. In order to address the non-convex scheduling optimization problem, global descent method is applied, which can continuously update the local optimal solutions by global descent function, and find global optimal solution. Finally, one modified IEEE 14-bus system is used to verify the performance of the proposed model and optimization solver.
\end{abstract}

\section{Introduction}

With the rapid development of economy, the shortage of energy resources have received extensive attention all over the world. Therefore, the development of renewable energy such as wind and solar power combined supply has become an important way to achieve energy-saving emission reduction strategy to target[1]. However, due to the random and intermittent characteristics of renewable energy, it brought huge challenge to the safety of power system operation. Therefore, the survey on the safe and economic operation of CCHP is significant.

In order to promote the long-term development of renewable energy, some incentives policies is needed. [2] analyses the dynamic effects of TGC and electric power market on the condition of TGC price is constant, [3] establish the equilibrium model of each power company benefits in the oligopoly conditions. However, these articles ignore the uncertainty brought by TGC price of renewable energy production and supply and demand. This paper studies optimization scheduling problems in the CCHP system of complementary energy based on green certificate trade.

\section{Mathematical model of CCHP system}

The conventional fossil energy conversion relationship in the CCHP unit is as follow:

$$
\begin{gathered}
P_{i, f o}=\frac{v_{i, f o}}{\omega} \eta_{i, f o, T P e} q_{i, f o} \\
H_{i, f o}=\frac{\left(1-v_{i, f o}\right)}{\omega} \eta_{i, f o, h} q_{i, f o}+\frac{v_{i, f o}}{\omega} \eta_{i, f o, T P h} q_{i, f o}
\end{gathered}
$$

Where, $i \in\{1,2, \mathrm{~K}, N\}$ represents CCHP unit, and $q_{i, f o}$ represents the fossil energy consumption of CCHP in the period of $\omega . \eta$ represents conversion efficiency. $P_{i, f o}$ and $H_{i, f o}$ represent electricity energy and heat energy production by CCHP. $v_{i, f o}$ represents scheduling factor.

The relationship between maximum output and primary energy is as follow:

$$
\begin{aligned}
P_{i, r e, s} & =f_{i, r e, T P e}\left(v_{i, r e} q_{i, r e}\right) \\
H_{i, r e, s} & =f_{i, r e, H}\left(\left(1-v_{i, r e}\right) q_{i, r e}\right)+f_{i, r e, T P h}\left(v_{i, r e} q_{i, r e}\right)
\end{aligned}
$$

Where, $P_{i, r e, s}$ and $H_{i, r e, s}$ represent maximum output. $q_{i, r e}$ represent the consumption of 
nonrenewable energy. $v_{i, r e}$ represents scheduling factor.

The relationship between the maximum power output and wind speed of wind turbine is as follow:

$$
P_{i, r e, s}=f_{i, w, P}\left(q_{i, w}\right)=\left\{\begin{array}{lc}
0 & q_{i, w}<q_{i, w, \text { in }}, q_{i, w}>q_{i, w, \text { out }} \\
k_{i, w} q_{i, w}+d_{i, w} & q_{i, w, \text { in }} \leq q_{i, w} \leq q_{i, w, \text { out }} \\
P_{i, w, r} & q_{i, w, r}<q_{i, w} \leq q_{i, w, \text { out }}
\end{array}\right.
$$

Where, $q_{i, w}$ represents wind speed. $q_{i, w, i n}, q_{i, w, r}, q_{i, w, o u t}$ represent cut-in speed, rated speed and cut-out speed. $P_{i, w, r}$ represents rated power of wind turbine. $k_{i, w}$ and $d_{i, w}$ represent linear coefficient.

The gain of green certificate trade in the CCHP can be expressed as:

$$
\pi_{t g c, i}=\varpi_{t g c} \cdot Q_{t g c, i}
$$

Where, $\varpi_{t g c}$ represent trading price of green certificate, $Q_{t g c, i}$ represents the quantity for sale green certificate.

The linear relationship between the green certificate trading price and market demand is as follow:

$$
\varpi_{t g c}=K_{0}+K_{1}\left[Q_{t g c, 0}-\sum_{i} Q_{t g c, i}\right]
$$

Optimal dispatch model of the objective function include production cost of fossil fuels and renewable energy production cost, overestimate/underestimate the punishment cost and green certificate trading cost. They can be represent by $\Pi_{1}, \Pi_{2}$ and $\Pi_{3}$.

$$
\underset{P_{i, r e}, P_{i, f o}, H_{i, r e}, H_{i, f o}}{\operatorname{Min}} E[\Pi]=\Pi_{1}+E\left[\Pi_{2}\right]+E\left[\Pi_{3}\right]
$$

Production costs include the cost of fuel and operation maintenance cost. They can be represented by $\Pi_{11}$ and $\Pi_{12}$.

$$
\begin{aligned}
& \Pi_{11}=\sum_{i} \sum_{f o} a_{i, f o, e} P_{i, f o}+b_{i, f o, e} P_{i, f o}^{2}+a_{i, f o, h}\left(H_{i, f o}-\theta_{i, f o} P_{i, f o}\right)+b_{i, f o, h}\left(H_{i, f o}-\theta_{i, f o} P_{i, f o}\right)^{2} \\
& \left.\Pi_{12}=\sum_{i} \sum_{f o}\left[c_{i, f o, e} P_{i, f o}+c_{i, f o, h}\left(H_{i, f o}-\theta_{i, f o} P_{i, f o}\right)\right]+\sum_{r e} \mid c_{i, r e, e} P_{i, r e}+c_{i, r e, h}\left(H_{i, r e}-\theta_{i, r e} P_{i, r e}\right)\right]
\end{aligned}
$$

Where, $\theta_{i, f o}, \theta_{i, r e}$ represent thermoelectric ratio of fossil and renewable energy unit. $a_{i, f_{o}}$ and $b_{i, f o}$ represent cost coefficient of fossil fuel. $c_{i, f o}$ and $c_{i, r e}$ represent cost coefficient of operation and maintenance.

Penalty cost can be expressed as:

$$
\Pi_{2}=\sum_{i} \sum_{r e} c_{i, r e, e}^{u}\left[P_{i, r e, s}-P_{i, r e}\right]^{+}+c_{i, r e, e}^{o}\left[P_{i, r e}-P_{i, r e, s}\right]^{+}+c_{i, r e, h}^{u}\left[H_{i, r e, s}-H_{i, r e}\right]^{+}+c_{i, r e, h}^{o}\left[H_{i, r e}-H_{i, r e, s}\right]^{+}
$$

Where, $c_{i, r e}^{u}$ and $c_{i, r e}^{o}$ represent underestimated and overestimated cost of penalty coefficient.

The trading cost of green certificate is as follow:

$$
\Pi_{3}=-\sum_{i} \pi_{t g c, i}=-\sum_{i} \varpi_{t g c} \cdot Q_{t g c, i}
$$

The power balance constraints between supply and demand is as follow:

$$
\left\{\begin{array}{l}
\sum_{i}\left(\sum_{f o} P_{i, f o}+\sum_{r e} P_{i, r e}\right)=\sum_{i} L_{i, e} \\
\sum_{i}\left(\sum_{f o} H_{i, f o}+\sum_{r e} H_{i, r e}\right)=\sum_{i} L_{i, h}
\end{array}\right.
$$

Where, $L_{i, e}$ and $L_{i, h}$ represent electrical load and cooling load.

The power system operation constraints is as follow:

$$
-P_{l}^{\max } \leq \sum_{i} \chi_{i l}\left(\sum_{f o} P_{i, f o}+\sum_{r e} P_{i, r e}-L_{i, e}\right) \leq P_{l}^{\max }
$$

The heat(cold) water pipe operation constraints is as follow: 


$$
\sum_{f o} H_{i, f o}+\sum_{r e} H_{i, r e}-L_{i, h}=C \rho A V_{i}\left(T_{i}-T_{i 0}\right)
$$

\section{Solving algorithm}

The optimization model above can be simplified to the following form:

$$
\begin{cases}\min & \sum_{i=1}^{N} f_{i}(x) \\ \text { s.t. } & A x \geq b \\ & E x=e\end{cases}
$$

Where, $A \in R^{m \times n}, \quad E \in R^{l \times n}, \quad b \in R^{m}, \quad c \in R^{l}, \quad x \in R^{n}, \quad \mathrm{D} \quad$ is feasible region, $D=\left\{x \in R^{n} \mid A x \geq b, E x=e\right\}$ 。

The specific steps of solving the optimization model are as follow:

1) Select calculation accuracy and initial feasible solution $x^{(0)}, k=0$;

2) $\mathrm{A}$ and $\mathrm{b}$ are divided into $A=\left[A_{1}, A_{2}\right]^{T}$ and $b=\left[b_{1}, b_{2}\right]^{T} \quad$ respectively; Where $A_{1}^{T} x^{(k)}=b_{1}, A_{2}^{T} x^{(k)}>b_{2}$;

3) If $M=\left[A_{1}, E\right]^{T}$ is null, then $d_{k}=-\nabla \sum_{i=1}^{n} f_{i}\left(x^{(k)}\right)$; or $d_{k}=-\left(I-M^{T}\left(M M^{T}\right)^{-1} M\right) \nabla \sum_{i=1}^{n} f_{i}\left(x^{(k)}\right)$; and if $\left\|d_{k}\right\| \leq \varepsilon$, stop counting, and $x^{(k)}$ is the optimal solution, otherwise, turn into the next step;

4) If $\lambda \geq 0, x^{(k)}$ is the optimal solution, otherwise, $d_{k}=-\widetilde{P} \nabla \sum_{i=1}^{n} f_{i}\left(x_{k}\right)$, turn into the next step;

5) Make one-dimensional linear search, and solve the optimal step size $\alpha_{k}$, and make

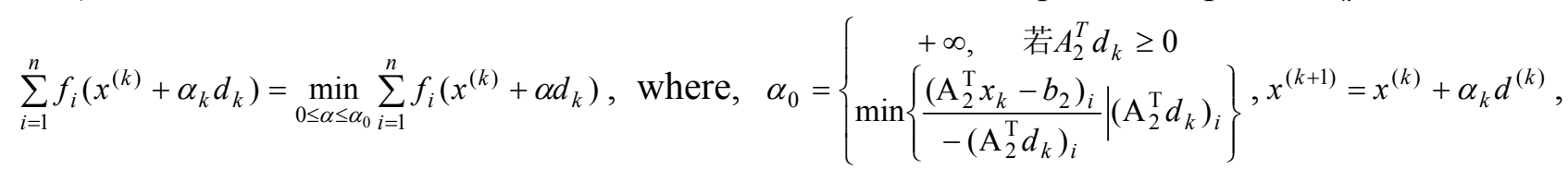
$k=k+1$, turn into step 2).

\section{Simulation}

This paper adopts IEEE-14 bus system as an example to analyze and calculate. Parameters of CCHP unit is show in Table 1, and the electricity and heat/cold energy demand is show in Table 2.

Tab.1 Parameters of CCHP unit

\begin{tabular}{ccccccccccc}
\hline \multirow{2}{*}{ node } & & $a$ & $b$ & $c$ & $c^{u}$ & $c^{o}$ & $\begin{array}{c}\text { Lower } \\
\text { limit }\end{array}$ & $\begin{array}{c}\text { Up } \\
\text { limit }\end{array}$ & $\eta$ & $\theta$ \\
\hline 1 & $\mathrm{H}$ & 12.1 & 0.03 & 23.1 & - & - & 0 & 100 & 0.5 & - \\
2 & $\mathrm{P}$ & - & - & 58.0 & 38 & 70 & 0 & 100 & - & - \\
3 & $\mathrm{P}$ & - & - & 60.0 & 38 & 70 & 0 & 100 & - & - \\
6 & $\mathrm{P}$ & 15.2 & 0.01 & 18.2 & - & - & 0 & 200 & 0.7 & 0.21 \\
& $\mathrm{H}$ & 14.4 & 0.02 & 21.3 & - & - & 0 & 200 & 0.6 & \\
8 & $\mathrm{P}$ & 15.5 & 0.02 & 19.2 & - & - & 0 & 100 & 0.6 & 0.23 \\
\hline
\end{tabular}

\begin{tabular}{cccccc}
\multicolumn{7}{c}{ Tab.2 } & The electricity and heat/cold energy demand \\
\hline \multirow{2}{*}{ node } & $\begin{array}{c}\text { Electric load } \\
(M W)\end{array}$ & $\begin{array}{c}\text { Cold/heat load } \\
(\mathrm{kJ})\end{array}$ & node & $\begin{array}{c}\text { Electric load } \\
(\mathrm{MW})\end{array}$ & $\begin{array}{c}\text { Cold/heat load } \\
(\mathrm{kJ})\end{array}$ \\
\hline 2 & 31.7 & 17.8 & 10 & 19.0 & 12.3 \\
3 & 21.2 & 15.5 & 11 & 16.5 & 11.1 \\
4 & 52.8 & 31.9 & 12 & 18.1 & 13.6 \\
5 & 20.6 & 14.2 & 13 & 23.5 & 15.0
\end{tabular}




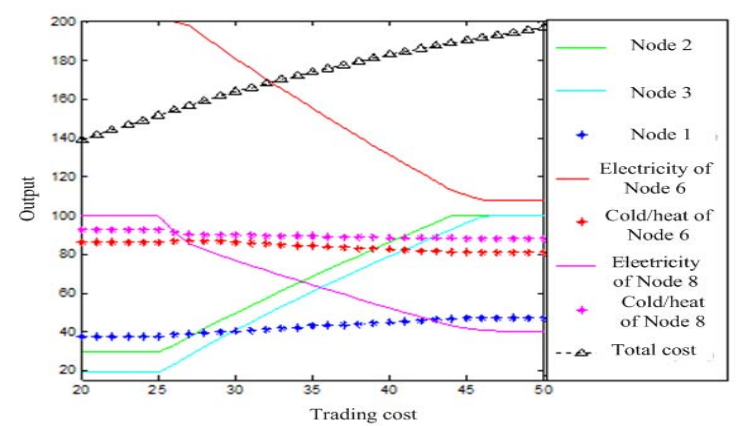

Fig.1 CCHP system optimal scheduling results

CCHP system optimal scheduling results is shown in Fig 1.As it is shown in Fig.1, when the green certificate trading price is very low, the output of two wind farms are low. With the increase of green certificate trading prices, when it exceeds $25 \$ /$ parts, the output of two wind farms approximately linearly increase. When it reach $25 \$ /$ parts, the output of two wind farms keep running at the rated condition. At the same time, node 6 and 8 cold/heat power reduced drastically, besides, the total cost of the system increase. As the effect of green certificate trading price change on conventional unit cooling and heating is very small, thus, node 1, 6 and 8 cooling and heating unit output with the change with transaction price slowly. Therefore, according to the actual situation, the choice of green certificate trading at the right price can reduce the cost of the system, and it will optimize the allocation of resources.

\section{Conclusion}

This paper proposes the multi energy complementary CCHP scheduling model based on green certificate trading and uses the global descend method for solving. The examples and simulation results show that the optimization model and its solution algorithm proposed are practical and effective. Therefore, the green certificate trading policies can stimulate the development and utilization of renewable energy sources. And it has a great effect on the optimal allocation of resources and reducing the operation cost of power system.

\section{References}

[1] Martin Geidl, Göran Andersson. Optimal Power Flow of Multiple Energy Carriers[J].IEEE Trans on Power Systems, 2007, 22(1): 145-155.

[2] Masoud Hasani-Marzooni, Seyed Hamid Hosseini. Dynamic Interactions of TGC and Electricity Markets to Promote Wind Capacity Investment[J].IEEE Systems Journal，2012，6(1): 46-57.

[3] Pedro Linares, Francisco Javier Santos, Mariano Ventosa, Luis Lapiedra.Incorporating oligopoly, CO2emissions trading and green certificates into a power generation expansion model[J].Automatica, 2008, 44(6): 1608-1620.

[4] A. Sheikhi, A.M. Ranjbar, F. Safe.A Novel Method to Determine the Best Size of CHP for an Energy Hub System[C].Proceedings of the 2nd International Conference on Electric Power and Energy Conversion Systems,Nov 15-17,2011, Sharjah, UAE: 1-7.

[5] Martin Geidl, Göran Andersson.Optimal Power Flow of Multiple Energy Carriers[J].IEEE Trans on Power Systems, 2007, 22(1): 145-155.

[6] M.Y. El-Sharkh, A. Rahman, M.S. Alam.Thermal Energy Management of a CHP Hybrid of Wind and a Grid-Parallel PEM Fuel Cell Power Plant[C].Proceedings of Power Systems Conference and Exposition, March 15-18, 2009, Seattle, WA: 1-6. 